\title{
Leptin receptor (Ob-R) mRNA expression and serum leptin concentration in patients with colorectal and metastatic colorectal cancer
}

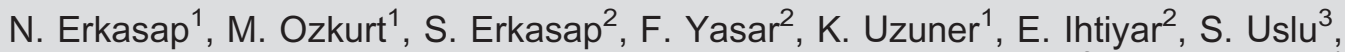 \\ M. Kara ${ }^{3}$ and O. Bolluk \\ ${ }^{1}$ Department of Physiology, Osmangazi University Medical Faculty, Meselik, Eskisehir, Turkey \\ ${ }^{2}$ Department of General Surgery, Osmangazi University Medical Faculty, Meselik, Eskisehir, Turkey \\ ${ }^{3}$ Department of Biochemistry, Osmangazi University Medical Faculty, Meselik, Eskisehir, Turkey \\ ${ }^{4}$ Department of Biostatistics, Osmangazi University Medical Faculty, Meselik, Eskisehir, Turkey
}

\begin{abstract}
The objective of the present study was to investigate the effect of leptin on the progression of colorectal carcinoma to metastatic disease by analyzing the serum leptin concentration and Ob-R gene expression in colon cancer tissues. Tissue samples were obtained from 31 patients who underwent surgical resection for colon (18 cases) and metastatic colon (13 cases) cancer. Serum leptin concentration was determined by an enzyme-linked immunosorbent assay (ELISA) and Ob-R mRNA expression by real-time polymerase chain reaction (RT-PCR) for both groups. ELISA data were analyzed by the Student $t$-test and RT-PCR data were analyzed by the Mann-Whitney U-test. RT-PCR results demonstrated that mRNA expression of Ob-R in human metastatic colorectal cancer was higher than in local colorectal cancer tissues. On the other hand, mean serum leptin concentration was significantly higher in local colorectal cancer patients compared to patients with metastatic colorectal cancer. The results of the present study suggest a role for leptin in the progression of colon cancer to metastatic disease without weight loss. In other words, significantly increased Ob-R mRNA expression and decreased serum leptin concentration in patients with metastatic colon cancer indicate that sensitization to leptin activity may be a major indicator of metastasis to the colon tissue and the determination of leptin concentration and leptin gene expression may be used to aid the diagnosis.
\end{abstract}

Key words: Leptin; Colorectal cancer; Metastatic colorectal cancer; Leptin receptor (Ob-R); Real-time PCR

\section{Introduction}

Colorectal cancer (CRC) is a commonly diagnosed cancer type. Adipose tissue dysfunction is likely to play a role in the growth of CRC (1). Leptin, the product of the ob/ob gene, is a $16-\mathrm{kDa}$ protein and an adipose tissuederived secreted protein that has been implicated primarily in the regulation of food intake as well as other metabolic parameters (2). Leptin, a protein hormone, promotes proliferation and differentiation of various cell types and is closely related to the development of cancer. Adipose tissue leptin mRNA and serum leptin concentration directly correlate with the amount of body fat, and there are many lines of evidence indicating that leptin is a signaling factor for the regulation of body weight (3). In addition, several in vitro studies have reported that leptin can act as a growth factor promoting the proliferation of cells and angiogenesis, and also has antiapoptotic tumorigenic effects on various cancer cell lines $(1,3-7)$.
Available clinicopathological data suggest that leptin and its receptor $\mathrm{Ob}-\mathrm{R}$ are expressed in cancer tissues and the binding of leptin to its receptor stimulates the proliferation of CRC cells (8). Similarly, studies of cellular models have also reported the involvement of leptin in colorectal carcinogenesis. For example, the proliferation and migration of both normal intestinal epithelial cells and CRC cells were stimulated by leptin (9).

Metastatic colorectal cancer (mCRC) is one of the leading causes of cancer-related death worldwide (10). In addition to CRC, $\mathrm{mCRC}$ is also commonly diagnosed. But, to our knowledge, there are no studies investigating serum leptin concentration and Ob-R mRNA expression in CRC and mCRC tissues concurrently. We, therefore, planned to analyze Ob-R mRNA expression by real-time (RT)-PCR as well as to measure the serum leptin concentration in both $\mathrm{CRC}$ and $\mathrm{mCRC}$ patients. 
It might be possible to improve the treatment and survival outcomes of the patients by earlier detection of $\mathrm{CRC}$ and particularly of $\mathrm{mCRC}$ by analyzing the Ob-R mRNA in the tissue or biopsy material. Therefore, we investigated leptin expression in human colorectal tissues in the sequential tumorigenic process including $\mathrm{CRC}$ and mCRC tissues.

\section{Material and Methods}

\section{Study populations}

The present study was approved by the Ethics Committee of Eskisehir Osmangazi University for Clinical Research (24/2009). Tissue specimens from 31 patients who underwent surgical resection for CRC (18 cases; 2 females, 16 males) and mCRC (13 cases; 3 females, 10 males) were collected within a 3-year period (from May 2008 to November 2011) at the Department of General Surgery, Hospital of Eskisehir Osmangazi University, Eskisehir, Turkey. Median age at diagnosis was 68 years (range: $46-81$ years) for CRC subjects and 70.3 years (range: $58-85$ years) for mCRC subjects. We statistically compared CRC and $\mathrm{mCRC}$ groups in terms of age, gender, body weight, height, and body mass index differences, and of the presence of diabetes, hyperlipidemia and other malignant diseases. There were no statistically significant differences in any of the clinical characteristics analyzed between groups. The clinical characteristics of the two patient groups are reported in Table 1.

\section{Tissue and serum samples}

Immediately after excision, tissue samples were fixed in RNAlater ${ }^{\circledR}$ solution (Life Technologies, USA) for the isolation of Ob-R mRNA gene expression. Blood samples were obtained at the time of operation; serum samples were obtained by centrifugation and stored at $-80^{\circ} \mathrm{C}$.

\section{RNA extraction and determination of Ob-R mRNA levels by RT-PCR}

The expression of Ob-R mRNA was examined in CRC and mCRC tissues. The mRNA levels of Ob-R (sense: $5^{\prime}-$ TGCACAGGAGCCAAGAGTGAA-3, anti-sense: 5' TGCACAGGAGCCAAGAGTGAA-3) in relation to the housekeeping gene, TATA-box binding protein (TBP) gene (sense: 5'-ATGCCACCAAACCTATG-3', antisense: 5'-GCTGTCTCATAATGTCCATTCG-3'), were determined by RT-PCR with TaqMan probes. RT-PCR thermal cycling conditions were as follows: $5 \mathrm{~min}$ at $65^{\circ} \mathrm{C}$, $60 \mathrm{~min}$ at $37^{\circ} \mathrm{C}$ for cDNA synthesis, $15 \mathrm{~s}$ at $95^{\circ} \mathrm{C}, 30 \mathrm{~s}$ at $65^{\circ} \mathrm{C}$ for 10 cycles, and $15 \mathrm{~s}$ at $72^{\circ} \mathrm{C}$. RT-PCR data were collected using the Rotor-gene 6000 detection system. Cycle threshold values were determined by automated threshold analysis. Primer quality (lack of primer-dimer amplification) was confirmed by melting curve analysis. Relative quantification of gene expression was performed
Table 1. Clinical characteristics of all participants of this study.

\begin{tabular}{lr}
\hline & Means \pm SD \\
\hline Age & \\
CRC & $64.67 \pm 10.42$ \\
mCRC & $65.0 \pm 10.10$ \\
Weight $(\mathrm{kg})$ & \\
CRC & $68.00 \pm 8.44$ \\
mCRC & $73.00 \pm 6.54$ \\
Height $(\mathrm{cm})$ & \\
CRC & $165.00 \pm 8.27$ \\
mCRC & $164.67 \pm 6.77$ \\
BMl (kg/m $\left.{ }^{2}\right)$ & \\
CRC & $24.9 \pm 1.25$ \\
mCRC & $26.98 \pm 2.56$ \\
Diabetes mellitus & \\
CRC & - \\
mCRC & - \\
Hyperlipidemia & \\
CRC & - \\
mCRC & - \\
Metastasis & \\
CRC & - \\
mCRC & Liver \\
\hline
\end{tabular}

Data for the CRC $(n=18)$ and mCRC $(n=13)$ groups were compared by the $t$-test. There were no statistically significant differences. $\mathrm{CRC}=$ colorectal cancer; $\mathrm{mCRC}=$ metastatic colorectal cancer; $\mathrm{BMI}=$ body mass index.

using the standard curve method, constructed with serial dilutions of control mRNA or RT-PCR amplicons. All experiments were carried out in triplicate. Ob- $R$ levels were standardized with TBP (ratio, Ob-R/TBP) to account for loading differences. Gene expression levels were reported using the median as a point estimator and the range of values.

\section{Determination of serum leptin concentration by ELISA}

Serum leptin concentration was determined using a commercially available human leptin ELISA kit (DIAsource ImmunoAssay, Belgium). The sensitivity of the assay was $0.04 \mathrm{ng} / \mathrm{mL}$ and inter-assay precision (coefficient of variation) was in the range of 10.2 to $12.7 \%$.

\section{Statistical analysis}

All statistical analyses were performed using SPSS 15.0 and SigmaStat 3.5. The descriptive statistisics are reported as $\mathrm{n}$ (sample size), mean and standard deviation for continuous variables and $n$ (sample size), median and 25th and 75th percentiles for categorical variables. Continuous normally distributed measurements were compared across the groups by the $t$-test. In the comparisons between the two groups, the continuous variables that did not show normal distribution were 
compared by the Mann-Whitney U-test. A $P$ value less than $0.05(P<0.05)$ was accepted as significant.

\section{Results}

mRNA expression of $\mathrm{Ob}-\mathrm{R}$ in human $\mathrm{mCRC}$ was significantly higher than in $C R C$ tissues $(P=0.016)$. The mean serum leptin concentration was significantly higher in CRC patients compared to $\mathrm{mCRC}$ patients $(P=0.024)$. Data for tissue leptin Ob-R mRNA expression and serum leptin concentration are reported in Table 2.

\section{Discussion}

The present study is the first to demonstrate that mRNA expression of Ob-R in human $\mathrm{mCRC}$ tissues is higher than that of $C R C$ tissues. On the other hand, serum leptin concentration was significantly higher in CRC than in $\mathrm{MCRC}$ patients.

Several in vitro studies have shown that leptin is a mitogenic, antiapoptotic and tumorigenic factor for different cancer cell lines (4-6). Koda et al. (9) showed that leptin and $\mathrm{Ob}-\mathrm{R}$ increase in tumoral tissue compared to nontumoral tissue in the breast and suggested that local, rather than endocrine, leptin might play a significant role in breast tumorigenesis. They also reported that leptin is overexpressed in human CRC cases as determined by immunohistochemistry and concluded that the hormone might contribute to CRC development and progression (9).

Although CRC is a very common type of cancer worldwide, a study comparing serum leptin concentration and expression of leptin receptors in cancer tissue in patients with $\mathrm{CRC}$ and $\mathrm{mCRC}$ has not been conducted before. However, there are conflicting reports about the role of leptin in the development of CRC. For example, Arpaci et al. (11) studied only serum leptin concentrations in patients with colon cancer and found them to be lower compared to healthy subjects, whereas Liu et al. (12) studied the effect of leptin on human colon cancer cell (HT29) growth and also the relationship between serum leptin concentration and colon cell proliferation. The

Table 2. Data for tissue leptin Ob-R mRNA expression and serum leptin concentration in colorectal cancer (CRC) and metastatic colorectal cancer ( $\mathrm{mCRC})$.

\begin{tabular}{lcc}
\hline & & $P$ \\
\hline Tissue leptin Ob-R mRNA & Median (25-75\%) & \\
expression & & \\
CRC & $0.72(0.38-1.34)$ & 0.016 \\
mCRC & $1.36(0.74-1.79)$ & \\
Serum leptin concentration & Mean \pm SD & \\
CRC & $4.02 \pm 2.26$ & 0.024 \\
mCRC & $1.45 \pm 0.94$ & \\
\hline
\end{tabular}

The Mann-Whitney U-test was used for statistical analysis. authors demonstrated that a high-fat diet results in enhancement of colon cell proliferation and carcinogenesis, which is mediated by increased serum leptin concentration (8).

In a study by Tutino et al. (13), leptin and leptin receptor concentration were only measured in serum and the authors suggested an association between leptin and $\mathrm{CRC}$ progression and aggressiveness. In an in vitro study by Attoub et al. (14), leptin promoted tumor invasion in CRC cell lines. However, Paik et al. (15) reported an inverse correlation between leptin expression and several prognostic parameters including tumor invasion depth, lymph node metastasis and lymphatic invasion. Uchiyama et al. (16) demonstrated that expression and activation of the long form leptin receptor (ObRL) were higher in colorectal adenoma and cancer tissues compared to the normal colorectal tissues. They also reported that leptin signaling might have a crucial role in the development of colorectal adenomas (16). However, Wiwanitkit (17) reported that interaction between leptin and leptin receptor has a critical role in the development of gastric carcinomas and leptin receptor suppresses the expression of leptin. Thus, the relationship of CRC and leptin was neither examined in terms of the involvement of leptin receptors in colorectal tissue nor compared to serum leptin concentration or leptin receptors in tumor tissue in mCRC patients. In patients with CRC or mCRC, the present study demonstrated an inverse correlation between serum leptin concentration and Ob-R mRNA gene expression, the latter being evaluated in colorectal tissue for the first time, rather than in serum. In other words, Ob-R mRNA gene expression in the tissues from $\mathrm{mCRC}$ patients was associated with decreased serum leptin concentration, suggesting an increased sensitivity to leptin receptors.

Leptin receptors are involved in colorectal tumor growth in vivo, indicating that leptin is an important factor for the regulation of CRC growth (1). Endo et al. (1) suggested that leptin is a crucial factor for the growth of CRC cells, regardless of dietary composition. Liu et al. (8) reported that leptin is overexpressed and binds to its receptor in pathological states, which results in tumor overgrowth in CRC. The authors also reported an association between the expression of leptin, leptin receptor, and vascular endothelial growth factor (VEGF) in $\mathrm{CRC}$, suggesting a possible role for the promotion of angiogenesis. They concluded that while binding of leptin to its receptor stimulates the proliferation of CRC cells, the synergism of leptin and VEGF can enhance the angiogenesis and promote cancer invasion and metastasis of colon cancers to other tissues in CRC patients (8). VEGF is a key regulator of angiogenesis (18) and a synergism was found between leptin and VEGF that results in the induction of blood vessel growth and increased vascular permeability (19). The proangiogenic and mitogenic effects of leptin have been implicated in the development 
and progression of various neoplasms. Studies have reported that leptin stimulates proliferation, migration, invasiveness, and survival of certain cancer cell types. Moreover, leptin may be also involved in tumor neoangiogenesis in several studies. Leptin can upregulate the expression of other angiogenic factors, such as basic fibroblast growth factor, interleukin 1- $\beta$, and leukemia inhibitory factor other than VEGF in cancer cells, which results in the potentiation of the proangiogenic effects of leptin. Because leptin and VEGF function synergistically (19), leptin may also contribute to tumor development in a similar manner as VEGF.

There are different studies, which measure serum leptin concentrations in CRC cases. Koda et al. (9) investigated whether leptin is expressed in colorectal tumors, and other clinicopathological parameters. However, we were the first to compare Ob-R mRNA expression and serum leptin concentration in CRC and $\mathrm{mCRC}$ patients. The results of the present study suggest that increased serum leptin concentration and decreased Ob-R mRNA expression in CRC might result in increased binding of leptin to its receptor, which can promote tumor growth. It is also a general pharmacological fact that whenever the first messenger is decreased then its receptor is increased (upregulation-downregulation). Moreover, Cao et al. (20) reported that decreased Ob-R expression might be related to the negative feedback resulting from high leptin protein concentrations.

The present study is the first to demonstrate that decreased serum leptin concentration is associated with increased Ob-R mRNA expression in the colon tissue of patients with $\mathrm{mCRC}$ in contrast to increased serum leptin concentration and decreased Ob-R mRNA expression in those with $\mathrm{CRC}$. Thus, we suggest that serum leptin

\section{References}

1. Endo H, Hosono K, Uchiyama $T$, Sakai E, Sugiyama M, Takahashi $H$, et al. Leptin acts as a growth factor for colorectal tumours at stages subsequent to tumour initiation in murine colon carcinogenesis. Gut 2011; 60: 1363-1371, doi: 10.1136/gut.2010.235754.

2. Erkasap N, Uzuner K, Serteser M, Koken T, Aydin Y. Gastroprotective effect of leptin on gastric mucosal injury induced by ischemia-reperfusion is related to gastric histamine content in rats. Peptides 2003; 24: 1181-1187, doi: 10.1016/j.peptides.2003.06.007.

3. Villarreal D, Reams G, Freeman RH, Taraben A. Renal effects of leptin in normotensive, hypertensive, and obese rats. Am J Physiol 1998; 275: R2056-R2060.

4. Hardwick JC, van den Brink GR, Offerhaus GJ, Van Deventer SJ, Peppelenbosch MP. Leptin is a growth factor for colonic epithelial cells. Gastroenterology 2001; 121: 7990, doi: 10.1053/gast.2001.25490.

5. Aparicio T, Kotelevets L, Tsocas A, Laigneau JP, Sobhani I, Chastre $\mathrm{E}$, et al. Leptin stimulates the proliferation of human colon cancer cells in vitro but does not promote the growth concentration and $\mathrm{Ob}-\mathrm{R}$ mRNA expression in colon tissue vary according to the stage of the colon cancer, such as local or metastatic disease.

Our results define a novel role for leptin signaling in the control of tumor growth in addition to its essential role in food intake and energy regulation. The role of leptin signaling is evidenced by the finding of increased $O b-R$ expression in $\mathrm{mCRC}$. The present study suggests that high levels of Ob-R mRNA expression in colorectal tissue may be indicative of metastasis. On the other hand, serum leptin concentration may have a greater effect on the progression of CRC than $\mathrm{mCRC}$ through the stimulation of blood vessel growth and vascular permeability, thus contributing to tumor neoangiogenesis.

Further studies of leptin will be helpful for a better understanding of $\mathrm{CRC}$ and $\mathrm{mCRC}$ development and it is possible that future chemotherapy regimens will include the potent inhibitors of leptin activity.

As a result, significantly increased Ob-R m-RNA gene expression and decreased serum leptin concentration in $\mathrm{mCRC}$ tissues might implicate that activation of leptin may play a role in the metastasis of CRC. In conclusion, we are the first to compare Ob-R mRNA expression and serum leptin concentration in CRC and $\mathrm{mCRC}$ patients. The results of the present study may indicate the presence of a biomarker for future studies that will evaluate the effectiveness of leptin and/or leptin receptor inhibitors for the treatment of CRC and $\mathrm{mCRC}$.

\section{Acknowledgments}

Research supported by a grant (\#200711018) from the Eskisehir Osmangazi University Scientific Research Projects Committee.

of colon cancer xenografts in nude mice or intestinal tumorigenesis in Apc(Min/+) mice. Gut 2005; 54: 11361145, doi: 10.1136/gut.2004.060533.

6. Rouet-Benzineb P, Aparicio T, Guilmeau S, Pouzet C, Descatoire V, Buyse $M$, et al. Leptin counteracts sodium butyrate-induced apoptosis in human colon cancer HT-29 cells via NF-kappaB signaling. J Biol Chem 2004; 279: 16495-16502, doi: 10.1074/jbc.M312999200.

7. Ishikawa M, Kitayama J, Nagawa H. Enhanced expression of leptin and leptin receptor (OB-R) in human breast cancer. Clin Cancer Res 2004; 10: 4325-4331, doi: 10.1158/10780432.CCR-03-0749.

8. Liu H, Wan D, Pan Z, Cao L, Wu X, Lu Z, et al. Expression and biological significance of leptin, leptin receptor, VEGF, and CD34 in colorectal carcinoma. Cell Biochem Biophys 2011; 60: 241-244, doi: 10.1007/s12013-010-9143-7.

9. Koda M, Sulkowska M, Kanczuga-Koda L, Surmacz E, Sulkowski S. Overexpression of the obesity hormone leptin in human colorectal cancer. J Clin Pathol 2007; 60: 902906, doi: 10.1136/jcp.2006.041004. 
10. Mao C, Liao RY, Qiu LX, Wang XW, Ding H, Chen Q. BRAF V600E mutation and resistance to anti-EGFR monoclonal antibodies in patients with metastatic colorectal cancer: a meta-analysis. Mol Biol Rep 2011; 38: 2219-2223, doi: 10.1007/s11033-010-0351-4.

11. Arpaci F, Yilmaz MI, Ozet A, Ayta H, Ozturk B, Komurcu S, et al. Low serum leptin level in colon cancer patients without significant weight loss. Tumori 2002; 88: 147-149.

12. Liu Z, Uesaka $\mathrm{T}$, Watanabe $\mathrm{H}$, Kato N. High fat diet enhances colonic cell proliferation and carcinogenesis in rats by elevating serum leptin. Int $J$ Oncol 2001; 19: 1009-1014.

13. Tutino V, Notarnicola M, Guerra V, Lorusso D, Caruso MG. Increased soluble leptin receptor levels are associated with advanced tumor stage in colorectal cancer patients. Anticancer Res 2011; 31: 3381-3383.

14. Attoub S, Noe V, Pirola L, Bruyneel E, Chastre E, Mareel M, et al. Leptin promotes invasiveness of kidney and colonic epithelial cells via phosphoinositide 3-kinase-, rho-, and racdependent signaling pathways. FASEB J 2000; 14: 23292338, doi: 10.1096/fj.00-0162.

15. Paik SS, Jang SM, Jang KS, Lee KH, Choi D, Jang SJ.
Leptin expression correlates with favorable clinicopathologic phenotype and better prognosis in colorectal adenocarcinoma. Ann Surg Oncol 2009; 16: 297-303, doi: 10.1245/ s10434-008-0221-7.

16. Uchiyama $T$, Takahashi $H$, Endo $H$, Sugiyama $M$, Sakai $E$, Hosono K, et al. Role of the long form leptin receptor and of the STAT3 signaling pathway in colorectal cancer progression. Int J Oncol 2011; 39: 935-940.

17. Wiwanitkit $\mathrm{V}$. Interaction between leptin and leptin receptor in gastric carcinoma: gene ontology analysis. Rev Esp Enferm Dig 2007; 99: 201-205.

18. Ray BK, Shakya A, Ray A. Vascular endothelial growth factor expression in arthritic joint is regulated by SAF-1 transcription factor. J Immunol 2007; 178: 1774-1782.

19. Ferla R, Bonomi M, Otvos L Jr, Surmacz E. Glioblastomaderived leptin induces tube formation and growth of endothelial cells: comparison with VEGF effects. BMC Cancer 2011; 11: 303, doi: 10.1186/1471-2407-11-303.

20. Cao Y, Gao F, Li CZ, Xue YM. Expression of leptin and its long-form receptor in the marginal cutaneous tissues of diabetic foot ulcers. Acta Diabetol 2012; 49 (Suppl 1): S205-S214, doi: 10.1007/s00592-012-0428-8. 